\title{
Theoretical and experimental study of the vibrational spectra of 1,5-dimethylcytosine
}

\author{
S.A. Brandán ${ }^{\text {a }, ~ G . ~ B e n z a l ~}{ }^{\text {a }}$, J.V. García-Ramos ${ }^{\text {, }}$, \\ J.C. Otero ${ }^{c}$, A. Ben Altabef ${ }^{\mathrm{a}, 1, *}$ \\ ${ }^{a}$ Instituto de Química Física, Facultad de Bioquímica, Química y Farmacia, Universidad Nacional de Tucumán, \\ San Lorenzo 456, 4000 Tucumán R., Argentina \\ ${ }^{\mathrm{b}}$ Instituto de Estructura de la Materia, Consejo Superior de Investigaciones Científicas, Serrano 121, 28006 Madrid, Spain \\ ${ }^{\mathrm{c}}$ Departamento de Química Física, Facultad de Ciencias, Universidad de Málaga, 29071 Málaga, Spain \\ Received 30 November 2005; received in revised form 10 October 2007; accepted 5 November 2007
}

\begin{abstract}
The Raman spectra of the solid 1,5-dimethylcytosine and the FTIR spectra at room and low temperatures respectively have been registered. Quantum mechanical calculations of energies, geometries and vibrational wavenumbers were carried out by using ab initio (HF) and Density Functional Theory (DFT/BLYP and B3LYP) methods with different basis sets. The best level of theory in order to reproduce the experimental wavenumbers is the BLYP method with the $6-31 \mathrm{G}^{*}$ basis set. The theoretical calculations indicate the presence of four stable tautomers of 1,5dimethylcytosine: amino-oxo; imino-oxo ( $\mathrm{a}$ and $\mathrm{b}$ ) and imino-hidroxy. Their geometries were optimised by using the BLYP/6-31G ${ }^{*}$ method, being the amino-oxo tautomer the most stable, followed by the imino-oxo tautomer, while the imino-hidroxy one is the most unstable. The complete assignment of the observed bands in the vibrational spectra of the amino-oxo tautomer is proposed in this work.
\end{abstract}

(C) 2007 Published by Elsevier B.V.

Keywords: Infrared; Raman; ab initio; DFT; 1,5-Dimethylcytosine

\section{Introduction}

The vibrational spectroscopy is a very important method for the study of the structure and properties of the biological systems [1-18]. For a few years new research efforts have been made to relate the biological functions of the constituent basis of nucleic acids, DNA and RNA [6-16], with the vibrational properties of uracil, adenine, guanine and cytosine and thiocytosine tautomers, since this relation allows an interpretation of its activity through its physicochemical properties.

DNA methylation plays important roles via regulation of numerous cellular mechanisms in diverse organisms, including humans [19-21]. The methylated derivatives of cytosine $[8,11,13]$ are very interesting due to the fact that the methylation process of a certain sequence of DNA bases can modulate the protein-DNA interaction and consequently

\footnotetext{
* Corresponding author. Tel.: +54 381 4311044; fax: +54 3814248169 .

E-mail address: altabef@fbqf.unt.edu.ar (A. Ben Altabef).

${ }^{1}$ Member of the Carrera del Investigador Científico, CONICET, R. Argentina.
}

regulate the gene function and the cell differentiation, as reported by Lapinski et al. [13]. For this reason the study of the vibrational spectra of 1,5-dimethylcytosine is very important in biological processes $[13,16]$.

Several spectroscopy studies of cytosine $[4,10,12,14]$ and thiocytosine tautomers [15], 1-methylcytosine [11] and 5methylcytosine $[13,16]$ have been performed, but only assignments of some bands in the spectra of 1,5-dimethylcytosine in the region between 800 and $100 \mathrm{~cm}^{-1}$ have been reported [8]. Moreover, a SERS study of 1,5-dimethylcytosine on $\mathrm{Ag}$ or $\mathrm{Cu}$ colloids has been reported $[17,18]$.

On the other hand, the vibrational assignments show the probable presence of more than one tautomeric form. It should be observed that in this type of systems it is possible to find different tautomers with similar stabilities and properties. In these cases, the interpretation of the spectra is more complex, especially at low temperatures, due to the overlapping of the bands of different tautomeric forms which complicates the assignment. For this reason it is very useful to have theoretical estimations of the wavenumbers of different tautomeric forms in order to recognize a particular tautomer in the spectra.

0924-2031/\$ - see front matter (C) 2007 Published by Elsevier B.V. doi:10.1016/j.vibspec.2007.11.001 

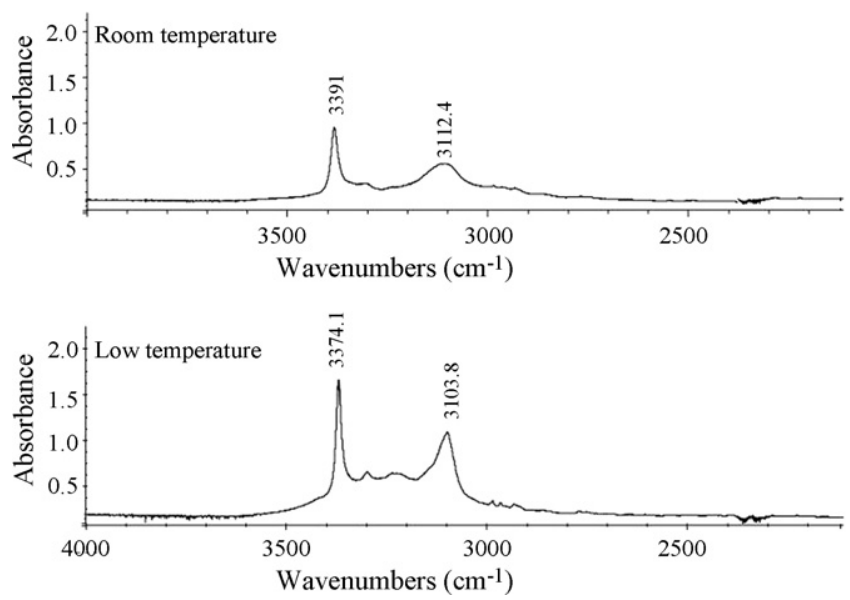

Fig. 1. The IR spectra of solid 1,5-dimethylcytosine at room temperature and at low temperature, between 4000 and $2000 \mathrm{~cm}^{-1}$.

\section{Experimental and theoretical calculations}

A pure Sigma commercial sample of 1,5-dimethylcytosine was used. The infrared spectra of 1,5-dimethylcytosine at room and low temperatures in $\mathrm{KBr}$ pellets, in the range between 4000 and $400 \mathrm{~cm}^{-1}$, were registered on a FTIR PerkinElmer model 1725-X spectrophotometer, equipped with a Globar source and DTGS detector. The infrared spectra at low wavenumbers were registered on a FTIR Digilab model 14 spectrophotometer using polyethylene pellets. Fig. 1 shows the infrared spectra of substance (at room and low temperature $(80 \mathrm{~K})$ in a RCII (VLT2) cell) between 4000 and $2000 \mathrm{~cm}^{-1}$, and Fig. 2 shows the $2000-400 \mathrm{~cm}^{-1}$ region. In addition, the Raman spectra of 1,5dimethylcytosine were registered in a glass capillary on a Jobin Yvon U-1000 double monocromador spectrometer, using an $\mathrm{Ar}^{+}$laser (Spectra Physics 165, $5145 \AA$ A exciting line) (Fig. 3).

All the calculations were made using the Gaussian 98 [22] program package. Geometries and force field calculations were carried out by using $a b$ initio (HF) and Density Functional
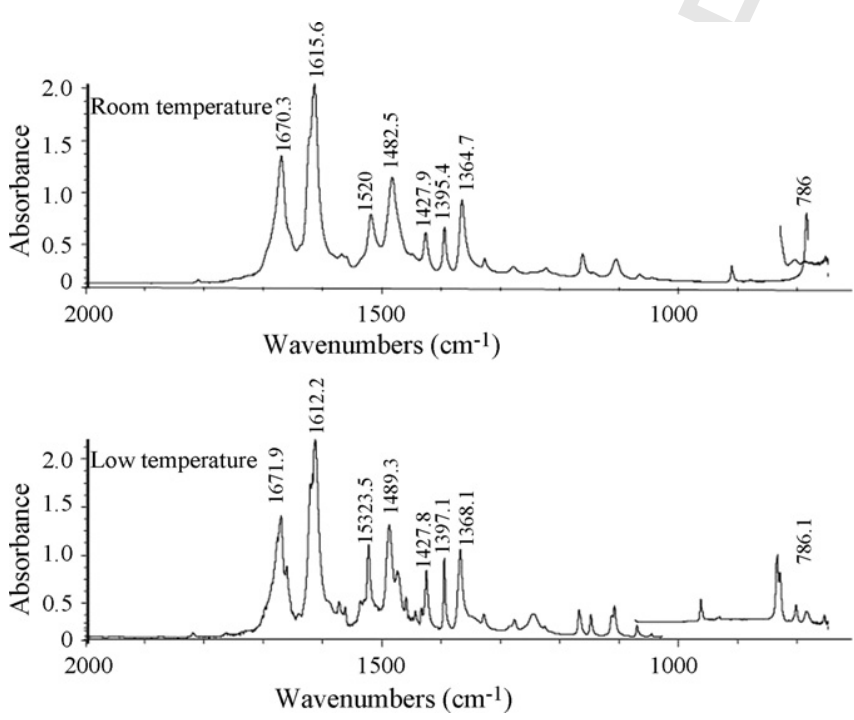

Fig. 2. The IR spectra of solid 1,5-dimethylcytosine at room temperature and at low temperature, between 2000 and $400 \mathrm{~cm}^{-1}$.

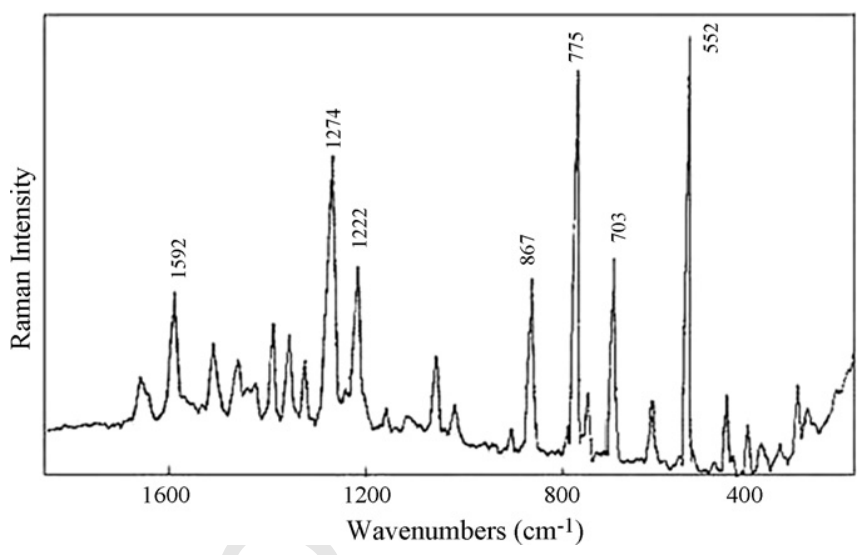

Fig. 3. Raman spectra of solid 1,5-dimethylcytosine.

Theory (DFT, BLYP and B3LYP) methods. The 3-21G, 6-31G, $6-31 G^{*}, 6-31 G^{* *}, 6-31+G, 6-31+G^{*}, 6-311 G^{*}, 6-311 G^{* *}, 6-$ $311+\mathrm{G}^{*}, 6-311+\mathrm{G}^{* *}, 6-311++\mathrm{G}^{* *}$ basis sets were used.

\section{Results and discussion}

From the analysis of the infrared spectra, especially at low temperatures, and the previous results on similar molecules such as cytosine [4,10,12,14] and 5-methylcytosine [13,16], four tautomers of 1,5-dimethylcytosine have been characterised with the aid of theoretical calculations: amino-oxo (I); iminooxo (IIa); imino-oxo (IIb) and imino-hidroxy (III). The corresponding structures are shown in Fig. 4. Their structures were optimised with different theoretical methods in order to obtain the relative stability, and the respective energies are shown in Table 1. As a result the amino-oxo tautomer is the most stable, followed by the imino-oxo forms; moreover, the imino-hidroxy structure shows a significant larger energy.

Nevertheless, experimental and calculated vibrational wavenumbers of the amino-oxo tautomer have been compared in order to select an adequate level of theory. For this purpose the statistical averages, standard deviations (RMSD) and reliability coefficients, were analysed (Table 2). The BLYP method shows smaller standard deviations and a better reliability coefficient [23] than the B3LYP method, while the $\mathrm{HF}$ results are poorer. The $6-31 \mathrm{G}^{*}$ basis set shows the best results and diffuse functions produce larger standard deviations. Therefore, the BLYP/6-31G ${ }^{*}$ with smaller $\operatorname{RMSD}\left(17.6 \mathrm{~cm}^{-1}\right)$ and better reliability coefficient $(0.99)$ has been selected for the optimisation of the geometry and the force field calculation of amino-oxo tautomer.

Besides, the interconversion between the tautomers of 1,5dimethylcytosine has been studied. The reaction paths connecting the structures I and III along the three transition states found (TS1, TS2 and TS3) can be noted as follows (see Fig. 5):

$\mathrm{I} \rightarrow \mathrm{TS} 1 \rightarrow \mathrm{IIa} \rightarrow \mathrm{TS} 2 \rightarrow \mathrm{IIb} \rightarrow \mathrm{TS} 3 \rightarrow \mathrm{III}$

The structures of the TS1, TS2 and TS3 transition states can be seen in Fig. 6. The BLYP/6-31G ${ }^{*}$ energies and the calculated 


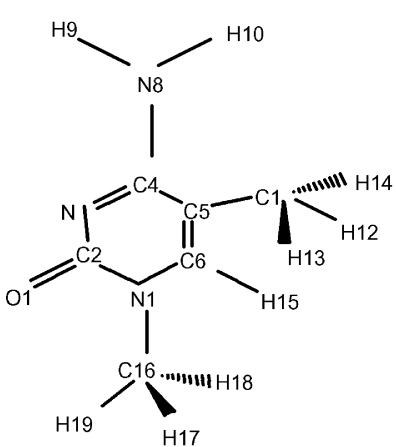

Amino-oxo (I)

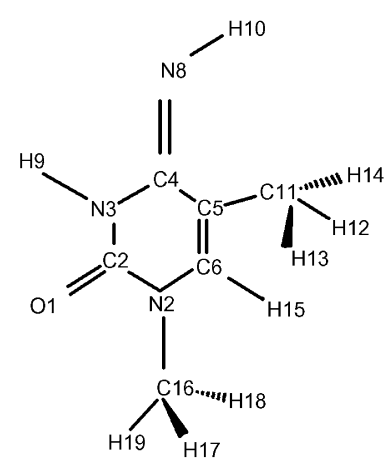

Imino-oxo (IIa)

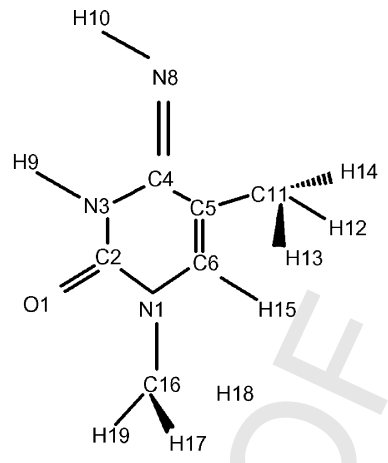

Imino-oxo (IIb)

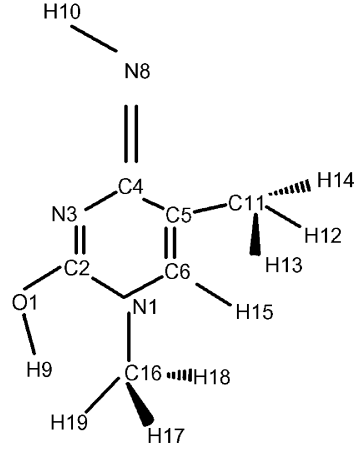

Imino-hidroxy (III)

Fig. 4. Tautomers of 1,5-dimethylcytosine and atom numbering.

dipolar moment of the different structures of 1,5-dimethylcytosine are presented in Table 3.

The I, IIa and IIb tautomers are energetically very close. They are the most stable, while the III tautomer has the highest relative energy $(29.1 \mathrm{kcal} / \mathrm{mol})$. Nevertheless, the higher value of the dipolar moment of structure III would justify its existence in spite of its larger relative energy. This same behaviour has been observed by Sambrano et al. [16] for the M4 tautomer of 5-methylcytosine. TS1 and TS3 transition states correspond with an intramolecular $\mathrm{H} 9$ transfer between $\mathrm{N} 8$ and $\mathrm{N} 3$ and $\mathrm{N} 3$ and $\mathrm{O} 7$, respectively. TS2 is associated with the out-of-plane deformation of the H10 atom of the imino-oxo tautomer. The normal mode of TS3 with imaginary frequency is related to the deformation of the $\mathrm{C} 2 \mathrm{O} 7 \mathrm{H} 9$ angle that connects the structures of the tautomeric forms IIb and III. The TS1 transition state connects the structures I and IIa, and is related to the deformation of the $\mathrm{C} 4 \mathrm{~N} 3 \mathrm{H} 9$ angle as well as to the $\mathrm{N} 8 \mathrm{H} 9$ stretching. The large relative energies of TS1 and TS3 (see

Table 1

Calculated B3LYP and BLYP energies (in Hartrees/molec.) for three tautomers of 1,5-dimethylcitosine

\begin{tabular}{|c|c|c|c|c|c|}
\hline \multirow[t]{2}{*}{ Methods } & \multirow[t]{2}{*}{ Basis } & \multicolumn{4}{|l|}{ Tautomers } \\
\hline & & Amino-oxo & Imino-oxo(a) & Imino-oxo(b) & Imino-hidroxy \\
\hline B3LYP & $\begin{array}{l}6-31 G \\
6-31 G^{*} \\
6-31+G^{*} \\
6-311 G^{* *}\end{array}$ & $\begin{array}{l}-473.41408 \\
-473.55845 \\
-473.57943 \\
-473.68262\end{array}$ & $\begin{array}{l}-473.41141 \\
-473.55621 \\
-473.57649 \\
-473.68649\end{array}$ & $\begin{array}{l}-473.40973 \\
-473.55461 \\
-473.57462 \\
-473.67918\end{array}$ & $\begin{array}{l}-473.35723 \\
-473.51153 \\
-473.53241 \\
-473.63880\end{array}$ \\
\hline BLYP & $\begin{array}{l}6-31 G \\
6-31 G^{*} \\
6-31+G^{*} \\
6-311 G^{* *}\end{array}$ & $\begin{array}{l}-473.25897 \\
-473.38852 \\
-473.41438 \\
-473.52658\end{array}$ & $\begin{array}{l}-473.25609 \\
-473.38610 \\
-473.41121 \\
-473.52526\end{array}$ & $\begin{array}{l}-473.25463 \\
-473.38467 \\
-473.40951 \\
-473.52373\end{array}$ & $\begin{array}{l}-473.20308 \\
-473.34214 \\
-473.36789 \\
-473.48265\end{array}$ \\
\hline
\end{tabular}

Table 2

Averages of the differences between experimental and theoretical vibrational frequencies at different levels of theory for the amino-oxo tautomer of 1,5 dimethylcitosine

\begin{tabular}{|c|c|c|c|c|c|c|c|c|c|c|c|}
\hline & $3-21 G^{*}$ & $6-31 \mathrm{G}$ & $6-31+\mathrm{G}$ & $6-31 G^{*}$ & $6-31+\mathrm{G}^{*}$ & $6-31++\mathrm{G}$ & $6-31++G^{*}$ & $6-311 \mathrm{G}$ & $6-311+\mathrm{G}$ & $6-311 \mathrm{G}^{*}$ & $6-311 \mathrm{G}^{* *}$ \\
\hline \multicolumn{12}{|l|}{ BLYP } \\
\hline Average & 35.6 & 35.6 & 34.4 & 30.5 & 30.6 & 34.5 & 30.7 & 34.5 & 34.7 & 30.8 & 32.6 \\
\hline RMSD & 20.8 & 24.9 & 22.8 & 17.6 & 18.3 & 22.8 & 18.3 & 21.7 & 22.4 & 19.5 & 19.5 \\
\hline Realibility coefficient & 0.99 & 0.99 & 0.99 & 0.99 & 0.99 & 0.99 & 0.99 & 0.99 & 0.99 & 0.99 & 0.99 \\
\hline \multicolumn{12}{|l|}{ B3LYP } \\
\hline Average & 51.6 & 54.6 & 50.8 & 50.7 & 49,74 & 52.7 & 48.6 & 46.3 & 44.5 & 46.9 & 40.1 \\
\hline RMSD & 44.6 & 55.6 & 53.9 & 49.5 & 56,62 & 54.7 & 47.4 & 48.1 & 46.4 & 46.8 & 37.7 \\
\hline Realibility coefficient & 0.99 & 0.99 & 0.99 & 0.99 & 0.99 & 0.99 & 0.99 & 0.99 & 0.99 & 0.99 & 0.99 \\
\hline \multicolumn{12}{|l|}{$\mathrm{HF}$} \\
\hline Average & 133.6 & 142.4 & 137.9 & 139.1 & 134.0 & 138.3 & 140.0 & 130.0 & 125.6 & 128.2 & 124.4 \\
\hline RMSD & 102.7 & 111.6 & 109.7 & 116.0 & 112.6 & 109.9 & 110.4 & 106.7 & 106.2 & 111.1 & 104.4 \\
\hline Realibility coefficient & 0.96 & 0.96 & 0.96 & 0.96 & 0.96 & 0.96 & 0.96 & 0.96 & 0.96 & 0.96 & 0.97 \\
\hline
\end{tabular}




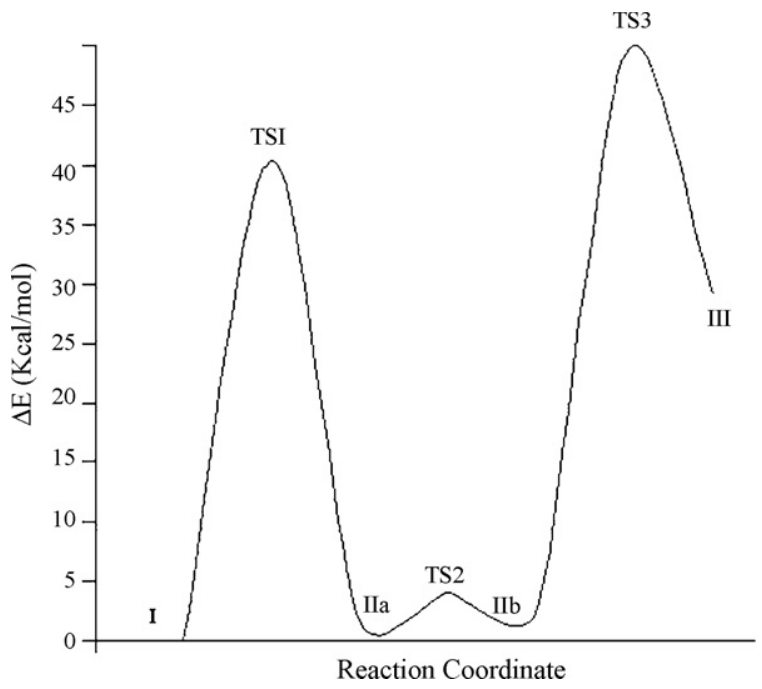

Fig. 5. Reaction path for the interconversion between the tautomers of 1,5dimethylcytosine.

Table 3 and Fig. 5) would prevent the conversion between the different tautomers. This fact would influence the tautomeric equilibrium by limiting the relative proportions of the iminooxo (IIa and IIb) and imino-hidroxy forms and, consequently, the relative intensities of the bands of the respective tautomers. The TS3 structure corresponds to a conformation of the iminohidroxy tautomer with the dihedral angle $\tau$; N1C2O7H9 equals $180^{\circ}$, and the imaginary wavenumber connects the imino-oxo IIb with the III forms.

The BLYP/6-31G ${ }^{*}$ optimised parameters of the four tautomers are presented in Table 4 together with the published data for 1-methylcitosine [24,25] and 5-methylcitosine [26,27]. There are no significant differences between the bond lengths and bond angles of the studied tautomers, except some ones related to the break of the $\mathrm{N} 3 \mathrm{C} 4$ double bond $(\mathrm{R} 3,4)$ when passing from the amino-oxo $(1.333 \AA)$ to the imino-oxo IIa $(1.423 \AA)$ forms, with the formation of C4N8 double bond
Table 3

$\mathrm{BLYP} / 6-31 \mathrm{G}^{*}$ total energies ( $E$; Hartree/molecule), relative energies ( $\Delta E ; \mathrm{kcal} /$ mol), and dipole moment ( $\mu$; Debye) for the tautomers and the transition states connecting the minima of 1,5-dimethylcitosine

\begin{tabular}{llrr}
\hline Structure & \multicolumn{1}{l}{$E$} & \multicolumn{1}{c}{$\Delta E$} & $\mu$ \\
\hline I & -473.38852 & 0.0 & 5.9 \\
TS1 & -473.32420 & 40.4 & 5.3 \\
IIa & -473.38610 & 1.5 & 4.6 \\
TS2 & -473.38211 & 4.0 & 2.4 \\
IIb & -473.38467 & 2.4 & 2.4 \\
TS3 & -473.31551 & 45.8 & 4.1 \\
III & -473.34215 & 29.1 & 7.2 \\
\hline
\end{tabular}

(R4,8) in the imino-oxo (1.298 $\AA$ ) and the imino-hidroxy $(1.301 \AA)$ tautomers; or with the $\mathrm{C} 2 \mathrm{O} 7$ double bond $(\mathrm{R} 2,7)$ in the amino-oxo form $(1.235 \AA$ ) that is a single bond $(1.374 \AA)$ in the imino-hidroxy tautomer. In the particular case of aminooxo, the values of the dihedral angles $\tau$; $(\mathrm{N} 3, \mathrm{C} 4, \mathrm{~N} 8, \mathrm{H} 9)$ and $\tau$; (N3, C4, N8, H10) equals $13.4^{\circ}$ and $156.3^{\circ}$, respectively, indicate that the two $\mathrm{H}$ atoms of the amino group are located on the same side and out of the ring plane. The torsion angles $\tau$; $(\mathrm{N} 8, \mathrm{C} 4, \mathrm{C} 5, \mathrm{C} 11)$ and $\tau ;(\mathrm{O} 7, \mathrm{C} 2, \mathrm{~N} 1, \mathrm{C} 16)$ are close to $0^{\circ}$ in almost all the tautomers. On the value of torsion angle $\tau$; (N1C2O7H9) in tautomer III is $13.8^{\circ}$ which indicates that the $\mathrm{H} 9$ atom is clearly displaced out of the ring plane.

\section{Vibrational spectrum}

1,5-Dimethylcytosine has 51 normal modes of vibration, all of them are active in infrared and Raman since the molecule does not possess any element of symmetry. The assignment of the vibrational spectra has been performed without taking into account possible intra and intermolecular interactions, on the basis of the BLYP/6-31G ${ }^{*}$ force field and the published assignments of cytosine [3,4,10,12-14], 1-methylcytosine [11], 5 -methylcytosine $[13,16]$ and related molecules like phenylsilane, toluene, benzonitrile, phenylacetylene and aniline [28-

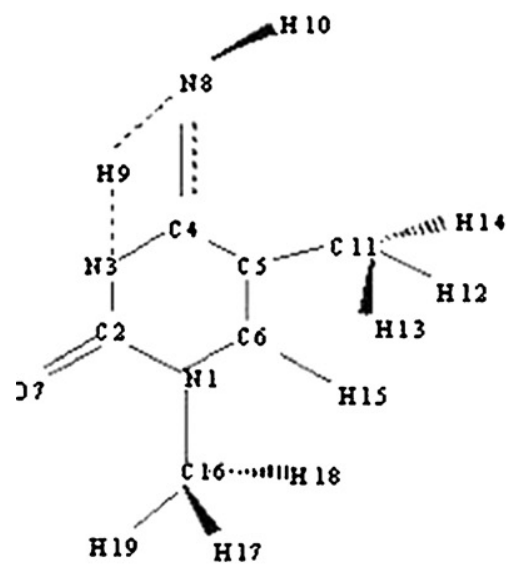

TSl
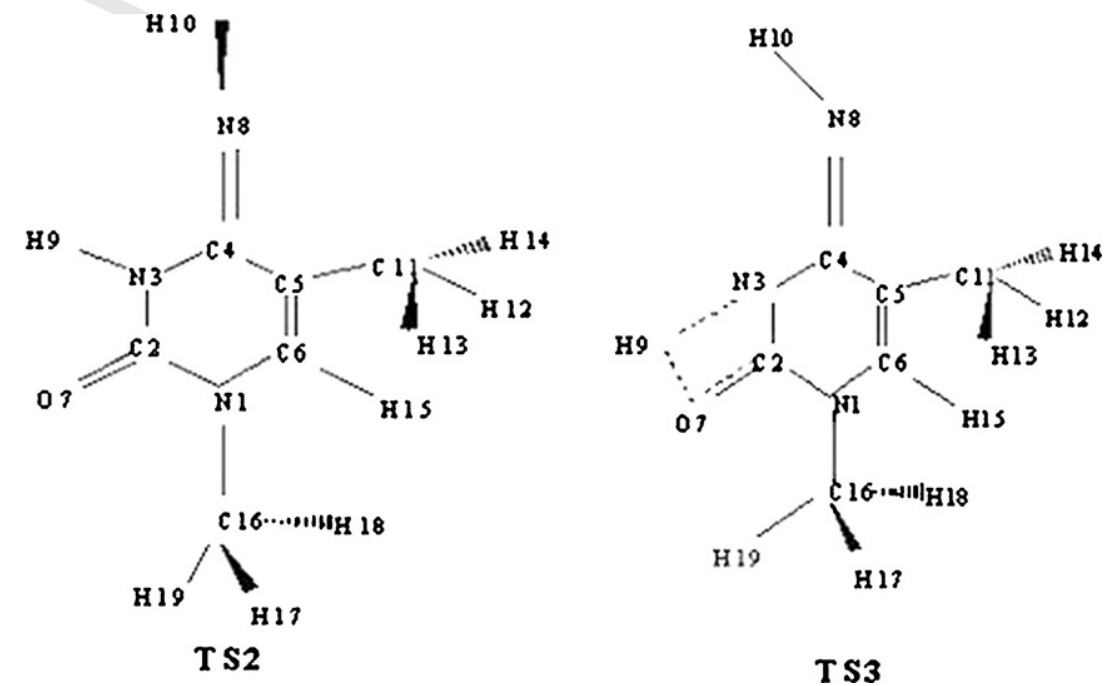

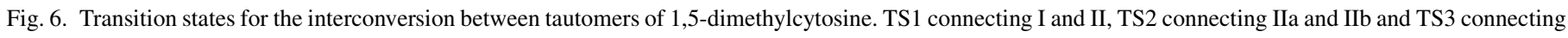
IIb and III tautomers, respectively (see Figs. 4 and 5). 
Table 4

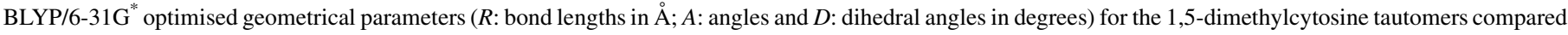
with the geometrical parameters of 1-methyl and 5-methyl cytosine

\begin{tabular}{|c|c|c|c|c|c|c|}
\hline Parameters & Amino-oxo & Imino-oxo IIa & Imino-oxo IIb & Imino-hidroxy III & 1-Methyl-cytosine [27] & 5-Methyl-cytosine [29] \\
\hline$R(1,2)$ & 1.459 & 1.408 & 1.418 & 1.399 & 1.395 & 1.376 \\
\hline$R(1,6)$ & 1.367 & 1.398 & 1.392 & 1.412 & 1.357 & 1.365 \\
\hline$R(1,16)$ & 1.472 & 1.473 & 1.472 & 1.469 & 1.464 & \\
\hline$R(2,3)$ & 1.380 & 1.401 & 1.390 & 1.294 & 1.358 & 1.354 \\
\hline$R(2,7)$ & 1.235 & 1.234 & 1.234 & 1.374 & 1.234 & 1.252 \\
\hline$R(3,4)$ & 1.332 & 1.423 & 1.417 & 1. 419 & 1.332 & 1.338 \\
\hline$R(3,9)$ & & 1.021 & 1.021 & & 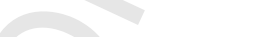 & \\
\hline$R(4,5)$ & 1.450 & 1.471 & 1.473 & 1.482 & 1.422 & 1.438 \\
\hline$R(4,8)$ & 1.384 & 1.298 & 1.298 & 1.301 & 1.336 & 1.337 \\
\hline$R(5,6)$ & 1.377 & 1.362 & 1.365 & 1.359 & 1.334 & 1.350 \\
\hline$R(5,11)$ & 1.514 & 1.510 & 1.512 & 1.509 & & 1.508 \\
\hline$R(6,15)$ & 1.094 & 1.092 & 1.093 & 1.092 & & \\
\hline$R(7,9)$ & & & & 0.980 & & \\
\hline$R(8,9)$ & 1.020 & & & & & \\
\hline$R(8,10)$ & 1.018 & 1.035 & 1.031 & 1.035 & & \\
\hline$R(11,12)$ & 1.102 & 1.102 & 1.101 & 1.103 & & \\
\hline$R(11,13)$ & 1.108 & 1.103 & 1.106 & 1.103 & & \\
\hline$R(11,14)$ & 1.106 & 1.103 & 1.106 & 1.103 & & \\
\hline$R(16,17)$ & 1.099 & 1.103 & 1.102 & 1.107 & & \\
\hline$R(16,18)$ & 1.101 & 1.103 & 1.102 & 1.098 & & \\
\hline$R(16,19)$ & 1.101 & 1.096 & 1.096 & 1.104 & & \\
\hline$A(2,1,6)$ & 121.2 & 121.3 & 121.2 & 116.4 & 120.1 & 121.3 \\
\hline$A(2,1,16)$ & 116.6 & 118.5 & 118.4 & 122.8 & 118.5 & \\
\hline$A(6,1,16)$ & 122.3 & 120.2 & 120.4 & 120.8 & 121.5 & \\
\hline$A(1,2,3)$ & 116.6 & 113.4 & 113.4 & 125.4 & 118.0 & 119.2 \\
\hline$A(1,2,7)$ & 117.9 & 124.1 & 123.3 & 117.2 & 118.6 & 119.0 \\
\hline$A(3,2,7)$ & 125.4 & 122.4 & 123.2 & 117.4 & 122.4 & 121.8 \\
\hline$A(2,3,4)$ & 120.6 & 128.8 & 129.1 & 120.1 & 120.0 & 119.5 \\
\hline$A(2,3,9)$ & & 112.8 & 115.2 & & & \\
\hline$A(4,3,9)$ & & 118.3 & 115.7 & 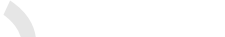 & & \\
\hline$A(3,4,5)$ & 124.5 & 113.4 & 113.6 & 117.4 & 121.8 & 123.1 \\
\hline$A(3,4,8)$ & 116.3 & 125.2 & 116.5 & 123.0 & 117.8 & 117.1 \\
\hline$A(5,4,8)$ & 119.1 & 121.3 & 129.8 & 119.5 & 120.4 & 119.9 \\
\hline$A(4,5,6)$ & 114.7 & 118.6 & 118.4 & 118.4 & 117.2 & 115.2 \\
\hline$A(4,5,11)$ & 122.8 & 118.6 & 119.4 & 119.2 & & 122.6 \\
\hline$A(6,5,11)$ & 122.4 & 122.8 & 122.2 & 122.4 & & 122.2 \\
\hline$A(1,6,5)$ & 122.2 & 124.3 & 124.3 & 122.2 & 121.8 & 121.7 \\
\hline$A(1,6,15)$ & 116.2 & 114.2 & 114.3 & 114.7 & & \\
\hline$A(5,6,15)$ & 121.6 & 121.4 & 121.4 & 123.1 & & \\
\hline$A(2,7,9)$ & & & & 110.6 & & \\
\hline$A(4,8,9)$ & 114.4 & & 2 & & & \\
\hline$A(4,8,10)$ & 118.9 & 111.2 & 109.9 & 107.9 & & \\
\hline$A(9,8,10)$ & 115.9 & & & & & \\
\hline$A(5,11,12)$ & 110.9 & 111.2 & 111.1 & 111.8 & & \\
\hline$A(5,11,13)$ & 112.3 & 110.8 & 111.6 & 110.4 & & \\
\hline$A(5,11,14)$ & 111.8 & 110.8 & 111.6 & 110.4 & & \\
\hline$A(12,11,13)$ & 106.9 & 108.8 & 107.7 & 109.0 & & \\
\hline$A(12,11,14)$ & 107.5 & 108.8 & 107.7 & 109.0 & & \\
\hline$A(13,11,14)$ & 107.0 & 106.2 & 106.9 & 105.9 & & \\
\hline$A(1,16,17)$ & 109.1 & 110.4 & 110.5 & 111.9 & & \\
\hline$A(1,16,18)$ & 109.9 & 110.4 & 110.5 & 108.7 & & \\
\hline$A(1,16,19)$ & 109.9 & 107.4 & 107.3 & 111.3 & & \\
\hline$A(17,16,18)$ & 110.0 & 109.2 & 109.2 & 107.4 & & \\
\hline$A(17,16,19)$ & 110.0 & 109.6 & 109.6 & 109.4 & & \\
\hline$A(18,16,19)$ & 107.7 & 109.6 & 109.6 & 107.9 & & \\
\hline$D(3,4,8,9)$ & 13.4 & -0.03 & & & & \\
\hline$D(3,4,8,10)$ & 156.3 & 0.008 & 179.9 & -0.4 & & \\
\hline$D(8,4,5,11)$ & 1.9 & -0.01 & -0.004 & 0.9 & & \\
\hline$D(7,2,1,16)$ & 0.004 & -0.01 & -0.011 & 2.9 & & \\
\hline
\end{tabular}


Table 5

BLYP/6-31G* calculated wavenumbers $\left(\mathrm{cm}^{-1}\right)$ and assignments of the calculated vibrational spectra of the tautomers of $1,5-$ dimethylcitosine

\begin{tabular}{|c|c|c|c|c|c|c|c|}
\hline \multicolumn{2}{|c|}{ Amino-oxo } & \multicolumn{2}{|c|}{ Imino-oxo IIa } & \multicolumn{2}{|c|}{ Imino-oxo IIb } & \multicolumn{2}{|c|}{ Imino-hidroxy } \\
\hline 3579.6 & $v_{\mathrm{s}} \mathrm{NH}_{2}$ & 3487.8 & $v \mathrm{~N} 3-\mathrm{H} 9$ & 3493.3 & $v \mathrm{~N} 3-\mathrm{H} 9$ & 3607.5 & $v$ O7-H9 \\
\hline 3463.1 & $\nu_{\mathrm{a}} \mathrm{NH}_{2}$ & 3298.5 & $v \mathrm{~N} 8-\mathrm{H} 10$ & 3362.1 & $v \mathrm{~N} 8-\mathrm{H} 10$ & 3303.1 & $v \mathrm{~N} 8-\mathrm{H} 10$ \\
\hline 3106.9 & $v$ C6-H15 & 3129.9 & $v$ C6-H15 & 3120.4 & $v$ C6-H15 & 3134.8 & $v$ C6-H15 \\
\hline 3074.9 & $\nu_{\mathrm{a}} \mathrm{C} 16-\mathrm{H}_{3}$ & 3110.6 & $\nu_{\mathrm{a}} \mathrm{C} 16-\mathrm{H}_{3}$ & 3111.1 & $v_{\mathrm{a}} \mathrm{C} 16-\mathrm{H}_{3}$ & 3074.3 & $\nu_{\mathrm{a}} \mathrm{C} 16-\mathrm{H}_{3}$ \\
\hline 3057.4 & $\nu_{\mathrm{a}} \mathrm{C} 16-\mathrm{H}_{3}$ & 3044.6 & $\nu_{\mathrm{a}} \mathrm{C} 11-\mathrm{H}_{3}$ & 3043.7 & $v_{\mathrm{a}} \mathrm{C} 11-\mathrm{H}_{3}$ & 3039.6 & $v_{\mathrm{a}} \mathrm{C} 11-\mathrm{H}_{3}$ \\
\hline 3033.6 & $\nu_{\mathrm{a}} \mathrm{C} 11-\mathrm{H}_{3}$ & 3026.7 & $\nu_{\mathrm{a}} \mathrm{C} 16-\mathrm{H}_{3}$ & 3027.4 & $v_{\mathrm{a}} \mathrm{C} 16-\mathrm{H}_{3}$ & 3025.9 & $v_{\mathrm{a}} \mathrm{C} 11-\mathrm{H}_{3}$ \\
\hline 2992.4 & $\nu_{\mathrm{s}} \mathrm{C} 16-\mathrm{H}_{3}$ & 3023.3 & $\nu_{\mathrm{a}} \mathrm{C} 11-\mathrm{H}_{3}$ & 2992.9 & $\nu_{\mathrm{a}} \mathrm{C} 11-\mathrm{H}_{3}$ & 2996.7 & $v_{\mathrm{a}} \mathrm{C} 16-\mathrm{H}_{3}$ \\
\hline 2981.5 & $\nu_{\mathrm{a}} \mathrm{C} 11-\mathrm{H}_{3}$ & 2975.0 & $v_{\mathrm{s}} \mathrm{C} 16-\mathrm{H}_{3}$ & 2975.3 & $\nu_{\mathrm{S}} \mathrm{C} 16-\mathrm{H}_{3}$ & 2971.1 & $\nu_{\mathrm{S}} \mathrm{C} 11-\mathrm{H}_{3}$ \\
\hline 2937.8 & $v_{\mathrm{s}} \mathrm{C} 11-\mathrm{H}_{3}$ & 2973.1 & $v_{\mathrm{s}} \mathrm{C} 11-\mathrm{H}_{3}$ & 2953.4 & $v_{\mathrm{s}} \mathrm{C} 11-\mathrm{H}_{3}$ & 2941.1 & $\nu_{\mathrm{S}} \mathrm{C} 16-\mathrm{H}_{3}$ \\
\hline 1714.2 & $v \mathrm{C} 2=07$ & 1730.6 & $v \mathrm{C} 2=07$ & 1736.2 & $v \mathrm{C} 2=\mathrm{O} 7$ & 1674.7 & $v \mathrm{C} 5=\mathrm{C} 6$ \\
\hline 1650.5 & $v \mathrm{C} 5=\mathrm{C} 6$ & 1661.2 & $v \mathrm{C} 5=\mathrm{C} 6$ & 1658.9 & $v \mathrm{C} 5=\mathrm{C} 6$ & 1614.6 & $v \mathrm{C} 2=\mathrm{N} 3$ \\
\hline 1610.3 & $\mathrm{\delta NH}_{2}$ & 1621.5 & $v \mathrm{C} 4=\mathrm{N} 8$ & 1606.9 & $v \mathrm{C} 4=\mathrm{N} 8$ & 1582.3 & $v \mathrm{C} 4=\mathrm{N} 8$ \\
\hline 1511.2 & $v \mathrm{~N} 3=\mathrm{C} 4$ & 1496.9 & $\delta_{\mathrm{a}} \mathrm{C} 16-\mathrm{H}_{3}$ & 1496.8 & $\delta_{\mathrm{a}} \mathrm{C} 16-\mathrm{H}_{3}$ & 1506.5 & $\delta_{\mathrm{a}} \mathrm{C} 16-\mathrm{H}_{3}$ \\
\hline 1496.2 & $\delta_{\mathrm{a}} \mathrm{C} 16-\mathrm{H}_{3}$ & 1493.7 & $\delta_{\mathrm{a}} \mathrm{C} 16-\mathrm{H}_{3}$ & 1494.3 & $\delta_{\mathrm{a}} \mathrm{C} 16-\mathrm{H}_{3}$ & 1482.3 & $\delta_{\mathrm{a}} \mathrm{C} 16-\mathrm{H}_{3}$ \\
\hline 1485.3 & $\delta_{\mathrm{a}} \mathrm{C} 11-\mathrm{H}_{3}$ & 1486.2 & $\delta_{\mathrm{a}} \mathrm{C} 11-\mathrm{H}_{3}$ & 1492.9 & $\delta_{\mathrm{a}} \mathrm{C} 11-\mathrm{H}_{3}$ & 1480.2 & $\delta_{\mathrm{a}} \mathrm{C} 11-\mathrm{H}_{3}$ \\
\hline 1474.8 & $\delta_{\mathrm{a}} \mathrm{C} 11-\mathrm{H}_{3}$ & 1461.5 & $\delta_{\mathrm{a}} \mathrm{C} 11-\mathrm{H}_{3}$ & 1470.1 & $\delta_{\mathrm{a}} \mathrm{C} 11-\mathrm{H}_{3}$ & 1456.6 & $\delta_{\mathrm{a}} \mathrm{C} 11-\mathrm{H}_{3}$ \\
\hline 1455.6 & $\delta_{\mathrm{a}} \mathrm{C} 16-\mathrm{H}_{3}$ & 1426.4 & $\delta_{\mathrm{s}} \mathrm{C} 16-\mathrm{H}_{3}$ & 1426.5 & $v \mathrm{C} 4-\mathrm{C} 5$ & 1450.6 & $\delta_{\mathrm{s}} \mathrm{C} 16-\mathrm{H}_{3}$ \\
\hline 1447.5 & $v \mathrm{C} 4-\mathrm{N} 8$ & 1416.3 & $v \mathrm{C} 4-\mathrm{C} 5$ & 1424.8 & $\delta_{\mathrm{s}} \mathrm{C} 16-\mathrm{H}_{3}$ & 1405.3 & $\delta_{\mathrm{s}} \mathrm{C} 11-\mathrm{H}_{3}$ \\
\hline 1431.9 & $\delta_{\mathrm{s}} \mathrm{C} 16-\mathrm{H}_{3}$ & 1410.0 & $\delta_{\mathrm{s}} \mathrm{C} 11-\mathrm{H}_{3}$ & 1409.4 & $\delta_{\mathrm{s}} \mathrm{C} 11-\mathrm{H}_{3}$ & 1398.8 & $v \mathrm{C} 4-\mathrm{C} 5$ \\
\hline 1405.4 & $\delta_{\mathrm{s}} \mathrm{C} 11-\mathrm{H}_{3}$ & 1380.2 & $\beta \mathrm{N} 3-\mathrm{H} 9$ & 1371.2 & $\beta \mathrm{N} 3-\mathrm{H} 9$ & 1357.6 & $v \mathrm{~N} 1-\mathrm{C} 2$ \\
\hline 1364.4 & $v \mathrm{~N} 1-\mathrm{C} 6$ & 1358.3 & $\beta$ C6-H15 & 1354.5 & $\beta$ C6-H15 & 1300.6 & $v \mathrm{C} 5-\mathrm{C} 11$ \\
\hline 1312.5 & $\beta$ C6-H15 & & & 1308.5 & $v$ N1-C6 & 1273.5 & $v \mathrm{~N} 1-\mathrm{C} 6$ \\
\hline 1229.5 & $v \mathrm{C} 5-\mathrm{C} 11$ & 1303.5 & $v$ N1-C6 & 1252.4 & $\beta \mathrm{N} 8-\mathrm{H} 10$ & 1224.2 & $v \mathrm{~N} 1-\mathrm{C} 16$ \\
\hline 1182.4 & $v \mathrm{C} 2-\mathrm{N} 3$ & 1230.8 & $v \mathrm{~N} 1-\mathrm{C} 16$ & 1216.5 & $v \mathrm{C} 2-\mathrm{N} 3$ & 1207.1 & $v \mathrm{C} 2-\mathrm{O} 7$ \\
\hline 1125.4 & $\rho \mathrm{C} 16-\mathrm{H}_{3}$ & 1202.9 & $v \mathrm{C} 2-\mathrm{N} 3$ & 1150.2 & $v \mathrm{C} 5-\mathrm{C} 11$ & 1130.8 & $\beta$ C6-H15 \\
\hline 1122.8 & $\rho \mathrm{C} 16-\mathrm{H}_{3}$ & 1143.1 & $\rho \mathrm{C} 16-\mathrm{H} 3$ & 1132.6 & $\rho \mathrm{C} 16-\mathrm{H} 3$ & 1114.4 & $\rho \mathrm{C} 16-\mathrm{H}_{3}$ \\
\hline 1055.5 & $\rho \mathrm{NH}_{2}$ & 1133.2 & $\rho \mathrm{C} 16-\mathrm{H} 3$ & 1094.9 & $v \mathrm{~N} 3=\mathrm{C} 4$ & 1075.5 & $v \mathrm{~N} 3=\mathrm{C} 4$ \\
\hline 1050.2 & $\rho \mathrm{C} 11-\mathrm{H}_{3}$ & 1091.9 & $v \mathrm{~N} 3=\mathrm{C} 4$ & 1051.1 & $\rho \mathrm{C} 11-\mathrm{H} 3$ & 1048.8 & $\rho \mathrm{C} 11-\mathrm{H}_{3}$ \\
\hline 1020.7 & $v \mathrm{~N} 1-\mathrm{C} 16$ & 1053.9 & $\rho \mathrm{C} 11-\mathrm{H} 3$ & 1041.8 & $\rho \mathrm{C} 16-\mathrm{H} 3$ & 1033.3 & $\rho \mathrm{C} 16-\mathrm{H}_{3}$ \\
\hline 1004.7 & $\rho \mathrm{C} 11-\mathrm{H}_{3}$ & 1043.2 & $v \mathrm{C} 5-\mathrm{C} 11$ & 999.3 & $\rho \mathrm{C} 11-\mathrm{H} 3$ & 999.2 & $\rho \mathrm{C} 11-\mathrm{H}_{3}$ \\
\hline 878.2 & $\gamma$ C6-H15 & 1003.7 & $\rho \mathrm{C} 11-\mathrm{H} 3$ & 864.6 & $\gamma$ C6-H15 & 868.3 & $\gamma \mathrm{N} 8-\mathrm{H} 10$ \\
\hline 812.9 & $v \mathrm{~N} 1-\mathrm{C} 2$ & 858.5 & $\gamma$ C6-H15 & 818.9 & $v \mathrm{~N} 1-\mathrm{C} 2$ & 838.7 & $\beta \mathrm{R} 1$ \\
\hline 736.4 & $\beta \mathrm{R}_{1}$ & 831.1 & $v \mathrm{~N} 1-\mathrm{C} 2$ & 774.1 & $\tau \mathrm{N} 8-\mathrm{H} 10$ & 814.2 & $\gamma$ C6-H15 \\
\hline 727.1 & $\gamma \mathrm{O} 7=\mathrm{C} 2$ & 793.9 & $\gamma \mathrm{N} 8-\mathrm{H} 10$ & 753.5 & $\beta \mathrm{R}_{1}$ & 733.4 & $\beta \mathrm{R}_{3}$ \\
\hline 711.3 & $\gamma \mathrm{N} 8-\mathrm{C} 4$ & 757.1 & $\beta \mathrm{R}_{1}$ & 704.4 & $\gamma \mathrm{N} 8-\mathrm{C} 4$ & 712.7 & $\gamma \mathrm{C} 4-\mathrm{N} 8$ \\
\hline 659.4 & $v \mathrm{C} 4-\mathrm{C} 5$ & 706.2 & $\gamma \mathrm{N} 8-\mathrm{C} 4$ & 697.9 & $\gamma \mathrm{O} 7=\mathrm{C} 2$ & 658.5 & $\beta \mathrm{R}_{2}$ \\
\hline 603.4 & $\beta C 2=07$ & 694.7 & $\gamma \mathrm{O} 7=\mathrm{C} 2$ & 675.1 & $\beta \mathrm{R}_{3}$ & 651.7 & $\gamma \mathrm{O} 7=\mathrm{C} 2$ \\
\hline 550.2 & $\gamma$ N8-H9 & 677.1 & $\beta \mathrm{R}_{3}$ & 643.4 & $\gamma \mathrm{N} 3-\mathrm{H} 9$ & 593.4 & $\beta \mathrm{C} 2=\mathrm{O} 7$ \\
\hline 521.8 & $\beta \mathrm{R}_{2}$ & 610.3 & $\beta C 2=07$ & 603.7 & $\beta \mathrm{C} 2=\mathrm{O} 7$ & 510.9 & $\tau \mathrm{R}_{1}$ \\
\hline 448.6 & $\beta R_{3}$ & 586.8 & $\gamma \mathrm{N} 3-\mathrm{H} 9$ & 515.5 & $\beta \mathrm{R}_{2}$ & 445.0 & $\tau \mathrm{R}_{3}$ \\
\hline 405.8 & $\tau \mathrm{R} 1$ & 504.6 & $\beta \mathrm{R}_{2}$ & 426.7 & $\tau \mathrm{R}_{1}$ & 400.6 & $\tau \mathrm{C} 4 \mathrm{~N} 8-\mathrm{H} 10$ \\
\hline 392.6 & $\gamma$ N8-H10 & 427.6 & $\tau \mathrm{R}_{1}$ & 390.6 & $\tau \mathrm{N} 3-\mathrm{H} 9$ & 365.8 & $\beta C 4-N 8$ \\
\hline 361.9 & $\beta \mathrm{C} 4-\mathrm{N} 8$ & 394.7 & $\tau \mathrm{N} 3-\mathrm{H} 9$ & 381.9 & $\beta \mathrm{C} 4-\mathrm{N} 8$ & 344.0 & $\beta \mathrm{N} 1-\mathrm{C} 16$ \\
\hline 319.3 & $\beta \mathrm{N} 1-\mathrm{C} 16$ & 378.6 & $\beta \mathrm{C} 4-\mathrm{N} 8$ & 333.2 & $\beta$ N1-C16 & 312.9 & $\gamma \mathrm{N} 1-\mathrm{C} 16$ \\
\hline 280.3 & $\gamma \mathrm{C} 11-\mathrm{C} 5$ & 330.9 & $\beta$ N1-C16 & 298.9 & $\gamma \mathrm{C} 5-\mathrm{C} 11$ & 276.9 & $\beta \mathrm{C} 5-\mathrm{C} 11$ \\
\hline 273.8 & $\beta \mathrm{C} 5-\mathrm{C} 11$ & 304.4 & $\gamma \mathrm{C} 5-\mathrm{C} 11$ & 277.6 & $\beta \mathrm{C} 5-\mathrm{C} 11$ & 236.8 & $\gamma \mathrm{O} 7-\mathrm{H} 9$ \\
\hline 225.2 & $\tau \mathrm{R}_{3}$ & 274.9 & $\beta$ C5-C11 & 189.6 & $\tau_{\mathrm{tw}} \mathrm{C} 11-\mathrm{H}_{3}$ & 192.9 & $\tau \mathrm{R}_{2}$ \\
\hline 188.3 & $\tau_{\mathrm{tw}} 11-\mathrm{H}_{3}$ & 186.6 & $\gamma \mathrm{N} 1-\mathrm{C} 16$ & 177.6 & $\gamma \mathrm{N} 1-\mathrm{C} 16$ & 173.7 & $\mathrm{~T}_{\text {wist }} \mathrm{C} 11-\mathrm{H}_{3}$ \\
\hline 155.7 & $\gamma \mathrm{N} 1-\mathrm{C} 16$ & 168.5 & $\tau_{\mathrm{tw}} \mathrm{C} 11-\mathrm{H}_{3}$ & 126.5 & $\tau \mathrm{R}_{3}$ & 116.6 & $\mathrm{~T}_{\text {wist }} \mathrm{C} 16-\mathrm{H}_{3}$ \\
\hline 101.9 & $\tau_{\mathrm{tw}} \mathrm{C} 16-\mathrm{H}_{3}$ & 125.2 & $\tau \mathrm{R}_{3}$ & 91.9 & $\tau \mathrm{R}_{2}$ & 101.3 & $\gamma \mathrm{C} 5-\mathrm{C} 11$ \\
\hline \multirow{2}{*}{77.3} & $\tau \mathrm{R}_{2}$ & 93.7 & $\tau \mathrm{R}_{2}$ & 64.5 & $\tau_{\mathrm{tw}} \mathrm{C} 16-\mathrm{H}_{3}$ & 46.1 & $\tau \mathrm{C} 16 \mathrm{~N} 1 \mathrm{C} 2 \mathrm{O} 7$ \\
\hline & & 72.4 & ${ }_{\mathrm{tw}} \mathrm{C} 16-\mathrm{H}_{3}$ & & & & \\
\hline
\end{tabular}

Abbreviations: $\nu$ : stretching, $\delta$ : bending, $\rho$ : rocking, $\gamma$ : wagging, $\tau$ : torsion, $\beta$ : in plane deformation, $\tau_{\mathrm{tw}}$ : twisting, $\beta_{\mathrm{R}}$ : deformation of the ring, $\tau_{\mathrm{R}}$ : torsion of the ring, a: antisymmetric, s: symmetric.

32]. The very close energies of the amino-oxo and the iminooxo, IIa and IIb forms, could indicate that all of them are present in the spectrum, which would complicate the discussion of the results. The calculated wavenumbers for the four tautomers of 1,5-dimethylcytosine (Table 5) have been compared with the experimental values. The wavenumbers of the bands registered in the infrared spectra at room and at low temperature and in the Raman spectra are shown in Table 6. Although the different isomers have many calculated wavenumbers with similar values, it can be verified that the aminooxo frequencies correspond better with the strongest and welldefined experimental bands. For this reason the assignments in
181

182 183 
Table 6

BLYP/6-31G ${ }^{*}$ wavenumbers $\left(\mathrm{cm}^{-1}\right)$ and assignments of the calculated vibrational spectra of tautomers of 1,5-dimethylcitosine

\begin{tabular}{|c|c|c|c|}
\hline $\begin{array}{l}\text { IR room } \\
\text { temperature }\end{array}$ & $\begin{array}{l}\text { IR low } \\
\text { temperature }\end{array}$ & RAMAN & Assignments \\
\hline $3546 \mathrm{sh}$ & $3418 \mathrm{sh}$ & $3539 \mathrm{vw}$ & $v_{\mathrm{a}} \mathrm{NH}_{2}$ \\
\hline 3391 vs & $3374.1 \mathrm{vs}$ & $3371 \mathrm{~m}$ & $v_{\mathrm{S}} \mathrm{NH}_{2}$ \\
\hline $3305.8 \mathrm{w}$ & $3302.9 \mathrm{w}$ & $3300 \mathrm{vvw}$ & $2 \times 919.7+1482.5=3304$ \\
\hline $3229 \mathrm{vw}$ & $3231.8 \mathrm{w}$ & $3267 \mathrm{vw}$ & $2 \times 1274+703=3251$ \\
\hline $3112.4 \mathrm{~m}$ & $3140 \mathrm{sh}$ & 3252 vvw & $2 \times 1615.6=3231.2$ \\
\hline $2990 \mathrm{vw}$ & $3103.8 \mathrm{~s}$ & & $4 \times 786=3144$ \\
\hline $2964.4 \mathrm{vw}$ & $2990 \mathrm{vw}$ & $3114 \mathrm{vw}$ & $v \mathrm{C} 6 \mathrm{H} 15$ \\
\hline 2933.1 & $2967.3 \mathrm{vw}$ & $3043 \mathrm{~m}$ & $v_{\mathrm{a}} \mathrm{C}_{16 \mathrm{H}_{3}}$ \\
\hline $2862.0 \mathrm{vvw}$ & 2935.9 & $3004 \mathrm{w}$ & $v_{\mathrm{a}} \mathrm{C} 16 \mathrm{H}_{3}$ \\
\hline 2765.3 & $2864.0 \mathrm{vvw}$ & $2978 \mathrm{~m}$ & $v_{\mathrm{a}} \mathrm{C} 11 \mathrm{H}_{3}$ \\
\hline 1813 vvw & $2773.8 \mathrm{vvw}$ & $2951 \mathrm{~m}$ & $v_{\mathrm{s}} \mathrm{C}_{16} \mathrm{H}_{3}$ \\
\hline $1670.3 \mathrm{~s}$ & $2756.7 \mathrm{vvw}$ & $2943 \mathrm{~m}$ & $v_{\mathrm{a}} \mathrm{C} 11 \mathrm{H}_{3}$ \\
\hline $1660 \mathrm{sh}$ & $1677.1 \mathrm{sh}$ & $2924 \mathrm{~s}$ & $v_{\mathrm{s}} \mathrm{C} 11 \mathrm{H}_{3}$ \\
\hline $1620.8 \mathrm{sh}$ & $1671.9 \mathrm{~s}$ & 2866 & $2 \times 1428=2856$ \\
\hline $1615.6 \mathrm{vs}$ & $1660 \mathrm{~m}$ & 2829 & $2 \times 703+1425=2831$ \\
\hline $1590 \mathrm{sh}$ & $1622.5 \mathrm{~s}$ & $1656 \mathrm{vw}$ & $1670.3+2 \times 552=2774.3$ \\
\hline $1571.3 \mathrm{vw}$ & $1612.2 \mathrm{vs}$ & $1592 \mathrm{~m}$ & $2 \times 703+1364.7=2770.7$ \\
\hline $1559.3 \mathrm{vw}$ & $1590 \mathrm{sh}$ & $1513 \mathrm{w}$ & $1274+552=1826$ \\
\hline $1520 \mathrm{~m}$ & $1571.3 \mathrm{w}$ & $1460 \mathrm{w}$ & $v \mathrm{C} 2=\mathrm{O} 7$ \\
\hline $1482.5 \mathrm{~s}$ & $1564.4 \mathrm{w}$ & $1425 \mathrm{w}$ & $v \mathrm{C} 5=\mathrm{C} 6$ \\
\hline $1464.4 \mathrm{sh}$ & $1550 \mathrm{vw}$ & $1391 \mathrm{~m}$ & $\delta \mathrm{NH}_{2}$ \\
\hline 1445 sh & $1523.5 \mathrm{~m}$ & $1357 \mathrm{w}$ & $?$ \\
\hline $1427.9 \mathrm{~m}$ & $1489.3 \mathrm{~s}$ & $1325 \mathrm{w}$ & $2 \times 786=1572$ \\
\hline $1395.4 \mathrm{~m}$ & $1475.7 \mathrm{w}$ & $1274 \mathrm{~s}$ & $\delta \mathrm{NH}_{2}$ \\
\hline $1364.7 \mathrm{~m}$ & $1460.3 \mathrm{w}$ & $1222 \mathrm{~m}$ & $2 \times 775=1550$ \\
\hline $1328.9 \mathrm{w}$ & $1451 \mathrm{vvw}$ & $1169 \mathrm{w}$ & $v \mathrm{~N} 3=\mathrm{C} 4$ \\
\hline $1281 \mathrm{vw}$ & $1445 \mathrm{vw}$ & $1060 \mathrm{w}$ & $v \mathrm{C} 4-\mathrm{N} 8$ \\
\hline $1274 \mathrm{sh}$ & $1434.7 \mathrm{vvw}$ & $1022 \mathrm{vw}$ & $\delta_{\mathrm{a}} \mathrm{C}_{16 \mathrm{H}_{3}}$ \\
\hline $1224.7 \mathrm{vw}$ & $1427.8 \mathrm{w}$ & 907 vw & $\delta_{\mathrm{a}} \mathrm{C} 11 \mathrm{H}_{3}$ \\
\hline $1163.3 \mathrm{w}$ & $1397.1 \mathrm{~s}$ & $867 \mathrm{~m}$ & $\delta_{\mathrm{a}} \mathrm{C} 11 \mathrm{H}_{3}$ \\
\hline $1147.9 \mathrm{sh}$ & $1368.1 \mathrm{~s}$ & $786 \mathrm{w}$ & $?$ \\
\hline $1106.9 \mathrm{w}$ & $1349.4 \mathrm{sh}$ & 775 vs & $\delta_{\mathrm{a}} \mathrm{C} 16 \mathrm{H}_{3}$ \\
\hline 1066 vvw & $1328.9 \mathrm{vw}$ & $750 w$ & $?$ \\
\hline $1045.5 \mathrm{vvw}$ & $1284.5 \mathrm{sh}$ & $703 \mathrm{~s}$ & $v \mathrm{C} 4-\mathrm{N} 8$ \\
\hline $910.7 \mathrm{w}$ & $1274.2 \mathrm{vw}$ & $624 \mathrm{w}$ & $\delta_{\mathrm{s}} \mathrm{C}_{16 \mathrm{H}_{3}}$ \\
\hline 878sh & $1245.2 \mathrm{vw}$ & 552 vs & $\delta_{\mathrm{s}} \mathrm{C} 11 \mathrm{H}_{3}$ \\
\hline $786 \mathrm{~m}$ & $1224.7 \mathrm{vw}$ & $541 \mathrm{sh}$ & $?$ \\
\hline 758.7 vvw & $1170.1 \mathrm{w}$ & $470 w$ & $v$ N1-C6 \\
\hline 741.7 vvw & $1147.9 \mathrm{w}$ & $457 \mathrm{vw}$ & $\beta$ С6H15 \\
\hline \multirow[t]{23}{*}{$705.8 \mathrm{vvw}$} & $1113.8 \mathrm{sh}$ & $427 \mathrm{w}$ & $v \mathrm{C} 5-\mathrm{C} 11$ \\
\hline & $1110.4 \mathrm{w}$ & $400 \mathrm{vw}$ & $703+552=1255$ \\
\hline & $1071.1 \mathrm{w}$ & $360 \mathrm{vw}$ & $v \mathrm{C} 2-\mathrm{N} 3$ \\
\hline & $1045.5 \mathrm{vvw}$ & $326 \mathrm{w}$ & $v \mathrm{C} 5-\mathrm{C} 11$ \\
\hline & $1026.7 \mathrm{vvw}$ & $304 \mathrm{w}$ & $\rho \mathrm{C}_{16} \mathrm{H}_{3}$ \\
\hline & $915.8 \mathrm{w}$ & 209 & $\rho \mathrm{Cl} 6 \mathrm{H}_{3}$ \\
\hline & $883.3 \mathrm{vvw}$ & 183 & $\rho \mathrm{NH}_{2}$ \\
\hline & $786.1 \mathrm{~m}$ & 165 & $\rho \mathrm{C} 11 \mathrm{H}_{3}$ \\
\hline & $779.2 \mathrm{w}$ & 139 & $v \mathrm{~N} 1-\mathrm{C} 16$ \\
\hline & $755.3 \mathrm{w}$ & 90 & $\rho \mathrm{C} 11 \mathrm{H}_{3}$ \\
\hline & $736.5 \mathrm{w}$ & & $\gamma \mathrm{C} 6 \mathrm{H} 15$ \\
\hline & $707.5 \mathrm{w}$ & & $v \mathrm{~N} 1-\mathrm{C} 2$ \\
\hline & & & $\beta \mathrm{R}_{1}$ \\
\hline & & & $v$ C4-C5 \\
\hline & & & $\gamma \mathrm{C} 2=\mathrm{O} 7$ \\
\hline & & & $\gamma \mathrm{C} 4-\mathrm{N} 8$ \\
\hline & & & $v \mathrm{C} 4-\mathrm{C} 5$ \\
\hline & & & $\gamma \mathrm{C} 4-\mathrm{N} 8$ \\
\hline & & & $\beta C 2=07$ \\
\hline & & & $\gamma \mathrm{N} 8 \mathrm{H} 9$ \\
\hline & & & $\beta R_{2}$ \\
\hline & & & $\beta R_{3}$ \\
\hline & & & $\tau \mathrm{R}_{1}$ \\
\hline
\end{tabular}

Table 6 (Continued)

\begin{tabular}{|c|c|c|}
\hline $\begin{array}{l}\text { IR room } \\
\text { temperature }\end{array}$ & $\begin{array}{ll}\text { IR low } & \text { RAMAN } \\
\text { temperature } & \end{array}$ & Assignments \\
\hline & & $\begin{array}{l}\gamma \mathrm{N} 8 \mathrm{H} 10 \\
\beta \mathrm{C} 4-\mathrm{N} 8 \\
\beta \mathrm{N} 1 \mathrm{C} 16 \\
\gamma \mathrm{C} 5 \mathrm{C} 11 \\
\beta \mathrm{C} 5 \mathrm{C} 11 \\
\tau \mathrm{R}_{3} \\
\tau_{\mathrm{tw}} \mathrm{C} 11 \mathrm{H}_{3} \\
\gamma \mathrm{N} 1 \mathrm{C} 16 \\
\tau_{\mathrm{tw}} \mathrm{C} 16 \mathrm{H}_{3} \\
\tau \mathrm{R}_{2}\end{array}$ \\
\hline
\end{tabular}

Abbreviations: $\nu$ : stretching, $\delta$ : bending, $\rho$ : rocking, $\gamma$ : wagging, $\tau$ : torsion, $\beta$ : in plane deformation, $\beta_{\mathrm{R}}$ : deformation of the ring, $\tau_{\mathrm{R}}$ : torsion of the ring, $\tau_{\mathrm{w}}$ : twisting, a: antisymmetric, s: symmetric, vs: very strong, s: strong, m: medium, w: weak, vw: very weak, vvw: very very weak, sh: shoulder.

Table 5 correspond with this isomer, which is the most stable as theoretical calculations predict. No evidences have been found of the significant presence of more than one tautomeric form.

\section{1. $4000-2000 \mathrm{~cm}^{-1}$ region}

In this region, the vibrational spectra show characteristic bands of normal modes related to the antisymmetric and symmetric stretchings of the $\mathrm{N}-\mathrm{H}$ and $\mathrm{C}-\mathrm{H}$ bonds of the amino and methyl groups, respectively, as well as the $\mathrm{C}-\mathrm{H}$ stretching modes of the ring.

The shoulder and the band recorded in the room temperature infrared spectrum at 3546 and $3391 \mathrm{~cm}^{-1}$, respectively, are assigned to antisymmetric and symmetric $\mathrm{NH}_{2}$ stretching respectively. In the Raman spectrum the strongest band is located at $3371 \mathrm{~cm}^{-1}$ and is assigned to the symmetric stretching mode. The calculated wavenumbers for 1,5dimethylcytosine and cytosine [3,4,10,14], 1-methylcytosine [11], 5-methylcytosine [13] and aniline [32] are in the same order. In this case the effect of anharmonicity is not so evident as in others molecules [33].

The broad, medium intensity band located at $3112.4 \mathrm{~cm}^{-1}$ in the room temperature infrared spectrum is recorded stronger at $3103.8 \mathrm{~cm}^{-1}$ in the spectrum at low temperature and is assigned to the C6-H15 stretching mode of the ring. In the Raman spectrum this last band is recorded very weak at $3114 \mathrm{~cm}^{-1}$.

The Raman bands at 3043, 3004, 2978, 2951, 2943 and $2924 \mathrm{~cm}^{-1}$ are assigned to antisymmetric and symmetric C-H stretching of both methyl groups. The order of the assignments is summarised in Table 6. In the Raman spectrum the strongest bands at 2951 and $2924 \mathrm{~cm}^{-1}$ are assigned to symmetric stretching modes corresponding to both $\mathrm{CH}_{3}$ groups.

\section{2. $1700-1500 \mathrm{~cm}^{-1}$ region}

In this region the $\mathrm{C}=\mathrm{OR}, \mathrm{C}=\mathrm{C}$ and $\mathrm{C}-\mathrm{N}$ stretching modes, as well as the $\mathrm{NH}_{2}$ deformation are expected. The characteristic strong infrared band recorded at $1670.3 \mathrm{~cm}^{-1}$ at room temperature is assigned to the $\mathrm{C} 2=\mathrm{O} 7$ stretching mode. This band is split in two bands at $1677.1 \mathrm{~cm}^{-1}$ and $1671.9 \mathrm{~cm}^{-1}$ in 
the spectrum at low temperature, like in cytosine [14] and 5methylcytosine [13]. This splitting could be attributed to a possible Fermi resonance as occurs in cytosine tautomers [12]. In the amino-oxo tautomer of 5-methylcytosine [13] this vibration is observed at $1735 \mathrm{~cm}^{-1}$ whereas in the same tautomer of cytosine is observed at 1719 and $1733 \mathrm{~cm}^{-1}$ [12].

The shoulder recorded in infrared at $1660 \mathrm{~cm}^{-1}$ shows a significant intensity, and is assigned to the C5=C6 stretching mode. The calculated wavenumber for this fundamental mode is $1650.5 \mathrm{~cm}^{-1}$.

The strong band at $1615.6 \mathrm{~cm}^{-1}$ is observed sharp and with stronger intensity in the low temperature spectrum, it being assigned to the $\mathrm{NH}_{2}$ deformation. This mode was observed at $1703 \mathrm{~cm}^{-1}$ [3], $1749 \mathrm{~cm}^{-1}$ [4], $1595 \mathrm{~cm}^{-1}$ [10], $1598 \mathrm{~cm}^{-1}$ [14] in amino-oxo tautomer of cytosine and at $1592 \mathrm{~cm}^{-1}$ in 1methylcytosine [11] and at $1595 \mathrm{~cm}^{-1}$ in 5-methylcytosine [13], while it is observed at $1618 \mathrm{~cm}^{-1}$ in the spectrum of aniline [32]. The calculated wavenumber for this deformation mode using HF/3-21G method is $1628 \mathrm{~cm}^{-1}$ in 5-methylcytosine [13] and $1579 \mathrm{~cm}^{-1}$ using CNDO/2 FORCE method in 1-methylcytosine [11]. In 1,5-dimethylcytosine this mode is calculated at $1610.3 \mathrm{~cm}^{-1}$. It is the strongest one of the Raman spectrum where it is recorded at $1592 \mathrm{~cm}^{-1}$. The shoulder at $1590 \mathrm{~cm}^{-1}$ in the infrared and Raman spectra remains unassigned. In the case of amino-oxo form of cytosine $[10,12]$ the band at $1599 \mathrm{~cm}^{-1}$ is assigned to the scissoring motion. In addition, splitting is observed in the spectrum at low temperature $\left(1571.3\right.$ and $\left.1564.4 \mathrm{~cm}^{-1}\right)$ for the band at room temperature $1571.3 \mathrm{~cm}^{-1}$ due to combinations in possible Fermi resonance with the fundamentals.

The medium intensity band recorded in the room temperature infrared spectrum at $1520 \mathrm{~cm}^{-1}$ and at $1523.5 \mathrm{~cm}^{-1}$ in the low temperature spectrum is assigned to the $\mathrm{N} 3=\mathrm{C} 4$ stretching.

\section{3. $1500-1000 \mathrm{~cm}^{-1}$ region}

The majority of the bands in this region are due to deformation modes of the $\mathrm{CH}_{3}$ groups and to stretching $\mathrm{C}-\mathrm{C}$ and $\mathrm{C}-\mathrm{N}$ bonds too. In this zone the theoretical calculations predict the frequencies and the intensities of the bands accurately for which permits to carry out a reliable assignment, as is observed in Fig. 7 compared with the experimental spectrum. Many of this bands appear split in two at low temperature (e.g. doublets: 1489.3 and 1475.7; 1460.3 and $1451 ; 1445$ and $1434.7 ; 1368.1$ and $1349.4 ; 1274.2$ and 1245.2; 1113.8 and 1110.4) due to Fermi resonance as in other aromatic ring [12]. According to their characteristic intensities, the strong band recorded at $1482.5 \mathrm{~cm}^{-1}$ in the room temperature spectrum and at 1489.3 and $1475.7 \mathrm{~cm}^{-1}$ at low temperature could be assigned to the $\mathrm{N}-\mathrm{CH}_{3}$ or $\mathrm{C}-\mathrm{CH}_{3}$ antisymmetric deformation. The shoulders at 1464.4 and $1445 \mathrm{~cm}^{-1}$ are assigned to the remaining $\mathrm{C}-\mathrm{CH}_{3}$ and $\mathrm{N}-\mathrm{CH}_{3}$ antisymmetric deformations, respectively. The band of medium intensity in the infrared spectrum at $1427.9 \mathrm{~cm}^{-1}$ is assigned to the $\mathrm{C} 4-\mathrm{N} 8$ stretching mode.

Force field calculations predict in this region the onset of stretching vibrations of the ring, in-plane $\mathrm{C}-\mathrm{H}$ deformation and

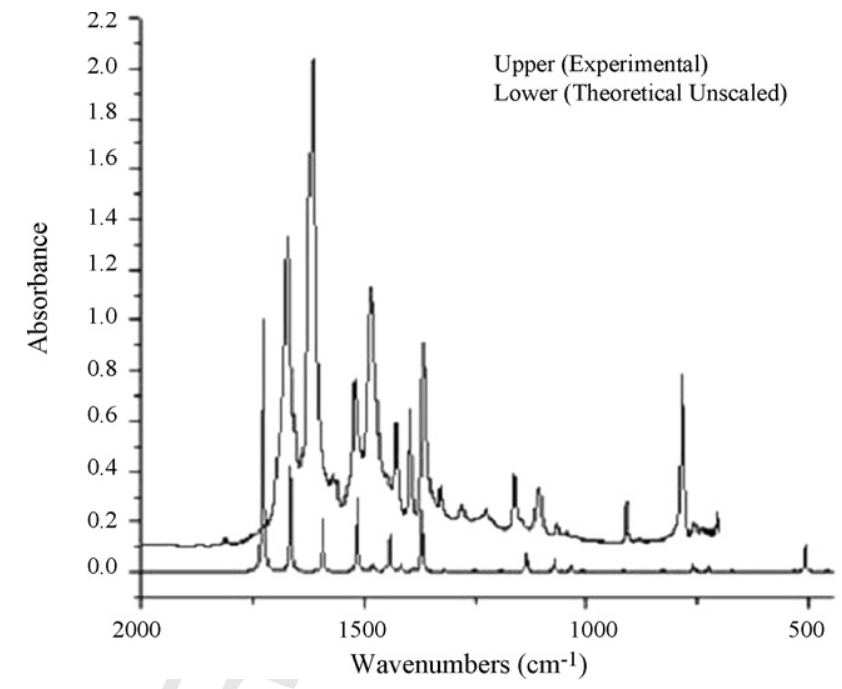

Fig. 7. The comparison IR spectra of solid 1,5-dimethylcytosine at room temperature with the theoretical spectrum between 2000 and $400 \mathrm{~cm}^{-1}$.

rocking modes of both $\mathrm{NH}_{2}$ and methyl groups. In similar molecules, like cytosine [3,4,12,14], 1-methylcytosine [11] and 5-methylcytosine [13], the same set of vibrations are observed.

The medium intensity band at $1395.4 \mathrm{~cm}^{-1}$ recorded in the infrared spectrum is assigned to the $\mathrm{N}-\mathrm{CH}_{3}$ symmetric deformation, while the band at $1364.7 \mathrm{~cm}^{-1}$ is assigned to $\mathrm{C}-\mathrm{CH}_{3}$ symmetrical deformation. The same band is observed in the Raman spectrum with very weak intensity at $1357 \mathrm{~cm}^{-1}$. The $1328.9 \mathrm{~cm}^{-1}$ band is assigned to N1-C6 stretching while the very weak band located at $1281 \mathrm{~cm}^{-1}$ is assigned to the C6H15 in-plane deformation. The theoretical spectrum predicts these modes at 1364.4 and $1312.5 \mathrm{~cm}^{-1}$, respectively (see Table 5) whereas the very weak band at $1224.7 \mathrm{~cm}^{-1}$ is assigned to the C2-N3 stretching.

The $\mathrm{N}-\mathrm{CH} 3$ rocking modes are assigned at 1163.3 and $1147.9 \mathrm{~cm}^{-1}$ infrared bands because the theoretical spectrum predicts these modes at 1125.4 and $1122.8 \mathrm{~cm}^{-1}$.

The $\mathrm{NH}_{2}$ rocking is observed in cytosine [14], 1methylcytosine [11] and 5-methylcytosine [13] at 1083, 1142 and $1074 \mathrm{~cm}^{-1}$, respectively. For this, the band at $1106.9 \mathrm{~cm}^{-1}$ of the room temperature spectrum, which appears split in 1113.8 and $1110.4 \mathrm{~cm}^{-1}$ at low temperature, is assigned to this vibration.

The $\mathrm{N}-\mathrm{CH}_{3}$ rocking in 1-methylcytosine [11] is observed at $1044 \mathrm{~cm}^{-1}$ while in the theoretical spectrum of 1,5-dimethylcytosine appear at $1055.5 \mathrm{~cm}^{-1}$ consequently the very weak band at $1066 \mathrm{~cm}^{-1}$ is assigned to this mode.

As observed in Table 5 the theoretical spectrum predicts the N1-C16 stretching of the amino-oxo tautomer at $1020.7 \mathrm{~cm}^{-1}$ therefore this mode is assigned to the very weak band at $1045.5 \mathrm{~cm}^{-1}$.

The band recorded at $1022 \mathrm{~cm}^{-1}$ in the Raman spectrum and at $1026.7 \mathrm{~cm}^{-1}$ at low temperature is assigned to the $\mathrm{C}-\mathrm{CH}_{3}$ rocking. This mode in the theoretical spectrum of 1,5dimethylcytosine is observed at $1004.7 \mathrm{~cm}^{-1}$ whereas in 5methylcytosine [13] with the HF/3-21G method appear theoretically hardly coupled with the $\mathrm{NH}_{2}$ rocking at 
$1074 \mathrm{~cm}^{-1}$ and with greater contribution at $1006 \mathrm{~cm}^{-1}$ but is observed experimentally at $998 \mathrm{~cm}^{-1}$.

\section{4. $1000-100 \mathrm{~cm}^{-1}$ region}

The assignments in this region are less reliable due to the large number of vibrations expected: ring deformations, $\mathrm{C} 2=\mathrm{O} 7, \mathrm{C} 4-\mathrm{N} 8, \mathrm{~N} 1-\mathrm{C} 16, \mathrm{C} 5-\mathrm{C} 11$ out-of-plane deformations and, ring torsions and $\mathrm{CH}_{3}$ and $\mathrm{NH}_{2}$ groups torsion.

The theoretical spectrum of the amino-oxo tautomer predicts the $\mathrm{C} 6 \mathrm{H} 15$ out-of-plane deformation at $878.2 \mathrm{~cm}^{-1}$ hence, the weak band observed in the spectrum at room temperature at $910.7 \mathrm{~cm}^{-1}$ is assigned to this vibrational mode.

The shoulder recorded at $878 \mathrm{~cm}^{-1}$ in the infrared, observed in the Raman spectrum as an intense band at $867 \mathrm{~cm}^{-1}$, is assigned to the $\mathrm{N} 1-\mathrm{C} 2$ stretching vibration. In the case of cytosine [14] this mode is calculated using $\mathrm{HF} / 6-31 \mathrm{G}^{* *}$ method with greater contribution at $911 \mathrm{~cm}^{-1}$ whereas in the aminooxo tautomer of 5-methylcytosine [13] appear theoretically with $\mathrm{HF} / 3-21 \mathrm{G}$ method at $886 \mathrm{~cm}^{-1}$ and observed at $877 \mathrm{~cm}^{-1}$.

The strongest infrared band of this region is observed at $786 \mathrm{~cm}^{-1}$, and it is split in two bands at 786.1 and $779.2 \mathrm{~cm}^{-1}$ in the spectrum at low temperature, while in the Raman spectrum only one band is observed at $775 \mathrm{~cm}^{-1}$. On the basis of both, the position and the intensity, it is assigned as a deformation of the ring, such mode being calculated at $736.4 \mathrm{~cm}^{-1}$. The remains deformation of the ring mode are observed at 541 and $470 \mathrm{~cm}^{-1}$. In related molecules like phenylsilane this modes appear at 704, 690 and $388 \mathrm{~cm}^{-1}$, in toluene at 789, 627 and $521 \mathrm{~cm}^{-1}$, in benzonitrile with greater contribution are observed at $997,752,623 \mathrm{~cm}^{-1}$ while in phenylacetylene at 998, 754 and $625 \mathrm{~cm}^{-1}$ and in aniline at 990 , 690 and $619 \mathrm{~cm}^{-1}$ [28-32]. The significative difference between this values probably is due at to calculations they carried out with different theoretical method.

The theoretical spectrum predicts the $\mathrm{C} 2=\mathrm{O} 7$ out-of-plane deformation at $727.1 \mathrm{~cm}^{-1}$. Thus, the very weak infrared band at $758.7 \mathrm{~cm}^{-1}$ is assigned to this vibration. In the amino-oxo tautomer of cytosine this mode is calculated at $794 \mathrm{~cm}^{-1}$ and is registered at $781 \mathrm{~cm}^{-1}$ [14]. The calculated wavenumber of the C4-N8 out-of-plane deformation, also called $\mathrm{NH}_{2}$ inversion mode [12], is $727.1 \mathrm{~cm}^{-1}$ and it is observed at $782 \mathrm{~cm}^{-1}$ in the Raman, while in the room and low temperature spectra it is recorded at $741.7 \mathrm{~cm}^{-1}$ and $736.5 \mathrm{~cm}^{-1}$, respectively.

The theoretical force field predicts the C4-C5 stretching mode at $659.4 \mathrm{~cm}^{-1}$. Thus, the strong Raman band at $703 \mathrm{~cm}^{-1}$ is assigned to this fundamental. It is calculated at $749 \mathrm{~cm}^{-1}$ and observed at $747 \mathrm{~cm}^{-1}$ in the amino-oxo tautomer of cytosine [14]. In the amino-oxo tautomer of 5-methylcytosine [13] is calculated at $886 \mathrm{~cm}^{-1}$ and observed at $877 \mathrm{~cm}^{-1}$.

The strong Raman band at $552 \mathrm{~cm}^{-1}$ is assigned as N8-H9 out-of-plane deformation. This mode is calculated at $565 \mathrm{~cm}^{-1}$ in the case of the amino-oxo tautomer of cytosine and observed at $525 \mathrm{~cm}^{-1}$ and $520 \mathrm{~cm}^{-1}$ in Argon and Neon matrices [12], respectively. This mode appear with greater contribution in the same tautomer of 5-methylcytosine [13] at $613 \mathrm{~cm}^{-1}$ and is observed at $609 \mathrm{~cm}^{-1}$.
The shoulder recorded in the Raman spectrum at $541 \mathrm{~cm}^{-1}$ is assigned as a ring deformation. In 5-methylcytosine this mode is observed at $478 \mathrm{~cm}^{-1}$ [13] while in the amino-oxo tautomer of cytosine is predicted at $526 \mathrm{~cm}^{-1}$ and observed at $535 \mathrm{~cm}^{-1}$ [14].

The very weak band in the Raman spectrum at $470 \mathrm{~cm}^{-1}$ is assigned to the deformation of the ring because the theoretical spectrum predicts a wavenumber of $448.7 \mathrm{~cm}^{-1}$.

The very weak Raman line of $457 \mathrm{~cm}^{-1}$ is assigned as a torsion of the ring. In the amino-oxo tautomer of 5methylcytosine this mode is calculated at $442 \mathrm{~cm}^{-1}$ [13] whereas at $405.8 \mathrm{~cm}^{-1}$ in 1,5-dimethylcytosine. In the case of the amino-oxo tautomer of cytosine it is predicted at $477 \mathrm{~cm}^{-1}$ and observed at $498 \mathrm{~cm}^{-1}$ [12].

The N8-H10 out-of-plane deformation is assigned to the weak Raman band recorded at $427 \mathrm{~cm}^{-1}$. It is calculated at $447 \mathrm{~cm}^{-1}$ in the amino-oxo tautomer of cytosine and observed at $535 \mathrm{~cm}^{-1}$ and $531 \mathrm{~cm}^{-1}$ in Argon and Neon matrices [12], respectively. In the amino-oxo tautomer of 5-methylcytosine it is calculated at $452 \mathrm{~cm}^{-1}$ and is observed at $406 \mathrm{~cm}^{-1}$ [13].

The assignments of the remaining bands in this region are shown in Table 6 and were carried out taking into account the calculated wavenumbers (see Table 5) and the assignments of the spectrum of the amino-oxo tautomers of cytosine [14] and 5-methylcytosine [13]. These bands are related to torsions of the ring and to the $\mathrm{N}-\mathrm{CH}_{3}$ and $\mathrm{C}-\mathrm{CH}_{3}$ out-of-plane modes.

\section{Conclusions}

Four tautomers of 1,5-dimethylcytosine have been theoretically found. According to the respective energies and the analysis of the vibrational spectra, the following stability order has been established: I $>$ IIa $>$ IIb $>$ III. The respective stability could depend strongly on their intermolecular interactions, temperature and aggregation state as previously observed in 5-methylcytosine [16].

The high value of the potential barrier would limit the quick interconversion between the imino-oxo IIa and IIb tautomers, although the simultaneous presence of both could be justified by the close values of energies. On the other hand, the high calculated barrier for the imino-hidroxy tautomer and its high energy discard to expect a significant abundance for this form.

The theoretical level that best reproduces the experimental vibrational wavenumbers of the amino-oxo tautomer is BLYP/ $6-31^{*}$ with a standard deviation of $17.6 \mathrm{~cm}^{-1}$ and a reliability coefficient of 0.99 .

The assignments of the vibrational modes of the amino-oxo (I) tautomer and the corresponding calculated wavenumbers for the four tautomers of 1,5-dimethylcytosine are reported. From the correspondence between the observed bands and the result of the calculated force field it was deduced that the most stable tautomer would be the amino-oxo (I) form.

\section{Acknowledgements}

S.A. Brandán thanks the Beca Banco Rio for a grant supporting this work. The research grants of CIUNT (Consejo 
de Investigaciones de la Universidad Nacional de Tucumán, R. Argentina) and CONICET (Consejo Nacional de Investigaciones Científicas y Técnicas, R. Argentina).

\section{References}

[1] R.J.H. Clark, R.E. Hester, Spectroscopy of Biological Systems, vol. 13, J. Wiley \& Sons, 1986.

[2] F. Korte (Ed.), Methodicum Chimicum, Vol. III Part I, Nucleic Acids, Proteins and Carbohydrates, Academic Press, New York, 1976.

[3] H. Susi, J.S. Ard, J.M. Purcell, Spectrochim. Acta 29A (1973) 725.

[4] E.D. Radchenko, G.G. Sheina, N.A. Smorygo, Y.P. Blagoi, J. Mol. Struct. 116 (1984) 387.

[5] M. Mathlöthi, A.M. Seuvre, J.L. Koening, Carboh. Res. 146 (1986) 1.

[6] M. Tsuboi, S. Takahashi, I. Harada, in: J. Duchesne (Ed.), Physico Chemical Properties of Nucleic Acids, vol. 2, Academic Press, New York, 1973.

[7] S. Aruna, G. Sahnmugam, J. Raman Spectrosc. 16 (4) (1985) 229.

[8] R. Letellier, M. Ghomi, E. Taillandier, Eur. Biophys. J. 14 (1987) 227.

[9] P. Lagant, P. Derreumaux, G. Vergoten, W. Peticolas, J. Comp. Chem. 12 (6) (1991) 731.

[10] M. Szczesniak, K. Szczepaniak, J.S. Kwiatkowski, K. KuBulat, W.B. Person, J. Am. Chem. Soc. 110 (1988) 8319.

[11] K. Kuczera, M. Szczesniak, K. Szczepaniak, J. Mol. Struct. 172 (1988) 73.

[12] M.J. Nowak, L. Lapinski, J. Fulara, Spectrochim. Acta 45A (2) (1989) 229.

[13] L. Lapinski, M.J. Nowak, J. Fulara, A. Lés, L. Adamowicz, J. Phys. Chem. 94 (1990) 6555.

[14] I.R. Gould, M.A. Vincent, I.H. Hillier, L. Lapinski, M.J. Nowak, Spectrochim. Acta 48A (6) (1992) 811.

[15] H. Rostkowska, M.J. Nowak, L. Lapinski, M. Bretner, T. Kulikowski, A. Lés, L. Adamowicz, Spectrochim. Acta 49A (4) (1993) 551.

[16] J.R. Sambrano, A.R. de Souza, J.J. Queralt, M. Oliva, J. Andrés, Chem. Phys. 264 (2001) 333.
[17] S. Sánchez-Cortes, J.V. García-Ramos, J. Raman Spectrosc. 21 (1990) 679.

[18] S. Sánchez-Cortes, J.V. García-Ramos, Langmuir 16 (2000) 764.

[19] G. Vilkaitis, I. Suetake, S. Klimasauskas, S. Tajima, J. Biol. Chem. 280 (1) (2005) 64.

[20] E. Merkiene, S. Klimasauskas, Nucleic Acids Res. 33 (1) (2005) 307.

[21] S. Klimasauskas, R. Gerasimaite, G. Vilkaitis, S. Kulakauskas, Nucleic Acids Symp. Ser. 2 (1) (2002) 73.

[22] M.J. Frisch, G.W. Trucks, H.B. Schlegel, G.E. Scuseria, M.A. Robb, J.R. Cheeseman, V.G. Zakrzewski, J.A. Montgomery Jr., R.E. Stratmann, J.C. Burant, S. Dapprich, J.M. Millam, A.D. Daniels, K.N. Kudin, M.C. Strain, O. Farkas, J. Tomasi, V. Barone, M. Cossi, R. Cammi, B. Mennucci, C. Pomelli, C. Adamo, S. Clifford, J. Ochterski, G.A. Petersson, P.Y. Ayala, Q. Cui, K. Morokuma, D.K. Malick, A.D. Rabuck, K. Raghavachari, J.B. Foresman, J. Cioslowski, J.V. Ortiz, A.G. Baboul, B.B. Stefanov, G. Liu, A. Liashenko, P. Piskorz, I. Komaromi, R. Gomperts, R.L. Martin, D.J. Fox, T. Keith, M.A. Al-Laham, C.Y. Peng, A. Nanayakkara, C. Gonzalez, M. Challacombe, P.M.W. Gill, B. Johnson, W. Chen, M.W. Wong, J.L. Andres, C. Gonzalez, M. Head-Gordon, E.S. Replogle, J.A. Pople, Program GAUSSIAN 98, revision A.7, Gaussian, Inc., Pittsburgh, PA, USA, 1998.

[23] P.S. Levy, S. Lemeshow, Sampling of Populations Methods and Applications, John Wiley \& Sons, Inc., New York, 1999.

[24] B. Trus, R. Marsh, Acta Cryst. B28 (1972) 1834.

[25] M. Rossi, T.J. Kistenmacher, Acta Cryst. B33 (1977) 3962.

[26] A. Takenaka, M. Kato, Y. Sasada, Bull. Chem. Soc. Jpn. 53 (1980) 383.

[27] C.T. Grainger, D. Bailey, Acta Cryst. B37 (1981) 1561.

[28] M. Alcolea Palafox, Recent Res. Dev. Phys. Chem. 2 (1998) 213.

[29] Y. Xie, J. Boggs, J. Comput. Chem. 7 (2) (1986) 158.

[30] A.G. Császár, G. Fogarasi, Spectrochim. Acta 45A (8) (1988) 845.

[31] A.G. Császár, G. Fogarasi, J.E. Boggs, J. Phys. Chem. 93 (1989) 7644.

[32] Z. Niu, K.M. Dunn, J.E. Boggs, Mol. Phys. 55 (2) (1985) 421.

[33] P. Pulay, G. Fogarasi, G. Pongor, J.E. Boggs, A. Vargha, J. Am. Chem. Soc. 105 (24) (1983) 7037. 